\title{
Hepatocellular carcinomas promote tumor-associated macrophage M2-polarization via increased B7-H3 expression
}

\author{
FU-BIAO KANG ${ }^{1,2^{*}}$, LING WANG $^{3^{*}}$, DONG LI $^{2 *}$, YIN-GE ZHANG ${ }^{2}$ and DIAN-XING SUN ${ }^{1,2}$ \\ ${ }^{1}$ Chinese PLA Medical School, Chinese PLA General Hospital, Beijing; ${ }^{2}$ Department of Liver Diseases, \\ Bethune International Peace Hospital, Shijiazhuang, Hebei; ${ }^{3}$ Cancer Research Institute, \\ The Fourth Hospital of Hebei Medical University, Shijiazhuang, Hebei, P.R. China
}

Received July 7, 2014; Accepted September 8, 2014

DOI: $10.3892 /$ or.2014.3587

\begin{abstract}
B7 family members are aberrantly expressed on the human hepatocellular carcinoma (HCC) cell surface, and induce local and systemic immunosuppression. Tumorassociated macrophages (TAMs) are a significant immune cell subpopulation in HCC and may be induced to express co-inhibitory molecules including B7 homologue 3 (B7-H3). In the present study, $79.3 \%$ of the HCC tissue samples showed high expression of B7-H3 which was positively correlated with the number of TAMs in the evaluated cancer tissues. Furthermore, high levels of TAMs or B7-H3 were associated with a shorter survival time of the HCC patients. In vitro, B7-H3 expression was upregulated at both the mRNA and protein levels in phorbol 12-myristate 13-acetate (PMA)-induced THP-1 cells cocultured with HepG2 cells in a Transwell system. In addition, B7-H3 promoted PMA-induced THP-1 cells to differentiate into the M2 phenotype, with evidence of increases in arginase 1 (Arg1), vascular endothelial cell growth factor (VEGF) and macrophage-derived chemokine (CCL22) mRNA following coculture with HepG2 cells. However, this phenomenon was abrogated through knockdown of B7-H3 by RNA interference or by blocking the signal transducer and activator of transcription 3 (STAT3) signaling pathway. Overall, these results suggest that the B7-H3-mediated STAT3 signaling pathway is an important mechanism for inducing M2-type polarization of TAMs, which accelerates HCC development. Our findings may support the development of novel therapeutic strategies for HCC patients through the skewing of the TAM phenotype by targeting the B7-H3 signaling pathway.
\end{abstract}

\section{Introduction}

Hepatocellular carcinoma (HCC), the most common type of primary liver malignancy, is the fifth most common cancer

Correspondence to: Professor Dian-Xing Sun, Chinese PLA Medical School, Chinese PLA General Hospital, Beijing, P.R. China

E-mail: sundianxing@hotmail.com

${ }^{*}$ Contributed equally

Key words: hepatocellular carcinoma, B7-H3, tumor-associated macrophages, STAT signaling pathway in men and the seventh most common cancer in women according to the International Agency for Research on Cancer (IARC) $(1,2)$. The incidence of HCC has increased dramatically by $80 \%$ in the last two decades in many of the developed and developing countries of the world (3). Despite better imaging studies and improvement of surgical techniques in this area, the 5-year survival rate for HCC still remains low and HCC is one of the malignancies with a high mortality rate $(4,5)$. Accumulating evidence suggests that immunotherapy is an alternative potent therapeutic strategy for patients with HCC $(6,7)$. Recent studies have shown that human cancer cells and immune cells in the cancer microenvironment upregulate expression of inhibitory B7 molecules and that these ectopic molecules contribute to tumor immune evasion (8). Therefore, the manipulation of the expression of and signaling through these molecules may be a promising strategy with which to treat human types of cancers.

In HCC, immunosuppressive ligands, including the costimulatory molecule B7 homologue 1 (B7-H1) and B7 homologue 3 (B7-H3), are aberrantly expressed at the tumor cell surface and in the cytoplasm (9-11). B7-H3, a newly identified member of the B7/CD28 superfamily, was identified as an accessory costimulatory molecule after initial antigen priming in cooperation with a putative counter receptor $(12,13)$. Although the role of B7-H3 in adaptive immune responses still remains controversial, overexpression of B7-H3 in HCC suggests that it may play a significant role in the 'immune escape' theory of tumors $(9,14)$. However, the multiple changes trigged by B7-H3 overexpression in HCC and how these overexpressed HCC cells disturb the microenvironment balance to induce immune escape remain to be clarified.

The tumor microenvironment is a complex system composed of many cell types, among which macrophages are the most abundant ones and its subpopulations can be recruited and polarized according to tumor-secreted cytokines in the tumor milieu, which have great potential for influencing tumor progression $(15,16)$. In general, the phenotype of these tumor-associated macrophages (TAMs) can be categorized into two subpopulations and each of them has diverse effects on the tumor: M1 macrophages are generally antitumoral, and M2 macrophages exert pro-tumoral effects $(17,18)$. In HCC, the phenotype of TAMs are largely immunosuppressive, and the degree of macrophage infiltration has been positively asso- 
ciated with both poor cancer-free survival and overall survival of patients (19).

B7-H3 is a multifunctional costimulatory molecule that is also involved in non-immunological functional regulation, such as cell growth, invasion and metastasis and drug resistance $(10,20,21)$. Recent studies have shown that $\mathrm{B} 7-\mathrm{H} 3$ proteins promote the progression of lung cancer by inducing the development of monocytes into M2 macrophages (22). In addition, B7-H3 augments proinflammatory cytokine release by binding its putative receptor on monocytes/macrophages and contributes positively to the development of sepsis (23). To date, substantial expression of B7-H3 in monocytes and tumor-infiltrating macrophages in HCC patients has not been documented; therefore, we aimed to denote the functions of B7-H3 in the present study.

\section{Materials and methods}

Patients and clinical specimens. According to the inclusion and exclusion criteria, 116 HCC tumor tissues and corresponding adjacent non-cancerous liver tissues used in immunohistochemical analysis were randomly obtained from patients undergoing liver curative resection between 2004 and 2008 who were hospitalized in the Department of Hepatobiliary Surgery, The Fourth Hospital of Hebei Medical University. The inclusion and exclusion criteria included patients: i) with distinctive pathologic diagnosis of $\mathrm{HCC}$; ii) with no anticancer treatment before liver resection; iii) who underwent primary and curative resection for HCC between 2004 and 2008; and iv) with complete clinicopathologic and follow-up data. All specimens were collected in the operating theater immediately ( $\leq 15 \mathrm{~min}$ ) after resection of the tumors and then were snap frozen in liquid nitrogen or fixed in $10 \%$ buffered formalin solution and embedded in paraffin for histological analysis. The histologic grade of tumor differentiation was determined by the Edmondson-Steiner grading system. Liver function was assessed by the Child-Pugh scoring system. Tumors were classified according to the WHO classification and the International Union against Cancer tumor-node-metastasis (TNM) classification. If patients had multiple lesions in the liver, we selected the main nodule for the present study. All samples were obtained following informed consent and their use was approved by the ethics committee of the institution. The median follow-up period was 33.5 months [range, 9-62 months; standard deviation (SD), 11.6 months]. At the last follow-up (December 31, 2012), 79 (68.1\%) patients were deceased, including 32 due to liver failure or bleeding from the gastrointestinal tract and the remaining 47 cases due to tumor recurrence.

Cell lines and culture conditions. The human monocytic cell line THP-1 and the HCC HepG2 cell line were obtained from the Cell Bank of the Chinese Academy of Sciences (Shanghai, China) and cultured according to the instructions from the American Type Culture Collection (ATCC). These cells were maintained in RPMI-1640 supplemented with $10 \%$ fetal calf serum (Gibco) and incubated at $37^{\circ} \mathrm{C}$ in a humidified chamber containing $5 \% \mathrm{CO}_{2}$.

Immunohistochemistry for B7-H3 and TAMs. Immunohistochemistry for B7-H3 was performed on a $5-\mu \mathrm{m}$ thick section using a two-step method with a polyclonal goat anti-B7-H3 antibody (1:200 dilution; R\&D Systems, Inc.) as the primary antibody and a rabbit anti-goat IgG antibody conjugated to horseradish peroxidase (ZSGB-BIO, Beijing, China) as the secondary antibody. Immunohistochemistry for TAMs was carried out on consecutive section using a threestep protocol with a monoclonal mouse anti-CD68 antibody (1:100 dilution; Abcam, UK) as the primary antibody and a rabbit anti-mouse IgG antibody conjugated to horseradish peroxidase (ZSGB-BIO) as the secondary antibody. In brief, paraffin sections were dewaxed in xylene and rehydrated through graded ethanol solutions. Antigens were retrieved by heating the tissue sections at $100^{\circ} \mathrm{C}$ for $30 \mathrm{~min}$ in EDTA solution. Sections were cooled down and immersed in $0.3 \% \mathrm{H}_{2} \mathrm{O}_{2}$ solution for $20 \mathrm{~min}$ to block endogenous peroxidase activity, and then rinsed in phosphate-buffered saline (PBS) for $5 \mathrm{~min}$, blocked with $5 \%$ BSA at room temperature for $20 \mathrm{~min}$, and incubated with primary antibodies at $4^{\circ} \mathrm{C}$ overnight. Negative controls were performed by replacing the specific primary antibody with PBS. After three PBS washes, sections were incubated with secondary antibodies for $30 \mathrm{~min}$ at room temperature. Diaminobenzene was used as the chromogen and hematoxylin as the nuclear counterstain. Sections were dehydrated, cleared and mounted.

Evaluation of B7-H3 and CD68 staining in cancer cells and TAMs were evaluated by authorized pathologists who had no knowledge of the patient clinical status and outcome. B7-H3 expression scores were given separately for the stained area and for the intensity of staining. Quantification was conducted as follows: $\leq 33 \%$ of the cancer cells, $1 ;>33$ to $\leq 66 \%$ of the cancer cells, $2 ;>66 \%$ of the cancer cells, 3 ; and intensity of staining: absent/weak, 1; moderate, 2; and strong, 3 . The intensity of B7-H3 staining was considered weak when either cytoplasmic expression or rare membranous condensation was present, moderate when incomplete and discontinuous moderate membranous expression was present, and strong when complete membranous expression of the molecule was present. Each section had a final grade that was derived from the multiplication of the area and intensity scores. Sections with a final score of $\leq 3$ were classified as tumors with low B7-H3 expression, whereas sections with a final score of $>3$ were classified as tumors with high B7-H3 expression.

To determine the intratumoral TAM densities, 10 representative high-power fields (x400 magnification) per tissue section were selected using a Leica DM2500 microscope. The number of nucleated cells with positive staining for CD68 in each of the examined cancer tissue areas was counted manually. The number of TAMs in each sample was determined by averaging the number of TAMs in at least 3 fields. According to the number of infiltration TAMs in the tumor, the average of grade I group was 75 , grade II group was 175 , and grade III group was 250 , respectively.

THP-1 cell and HCC cell coculture. Phorbol 12-myristate 13-acetate (PMA) (Sigma, St. Louis, MO, USA) was used to induce THP-1 cells to differentiate into macrophages (24). THP-1 cells $\left(1 \times 10^{6}\right)$ were seeded into the lower insert of a 6-well Transwell apparatus with a $0.4-\mu \mathrm{m}$ pore size and treated with PMA at a concentration of $350 \mathrm{nM}$ for $24 \mathrm{~h}$. After a thorough wash to remove all PMA, HepG2 cells were plated 


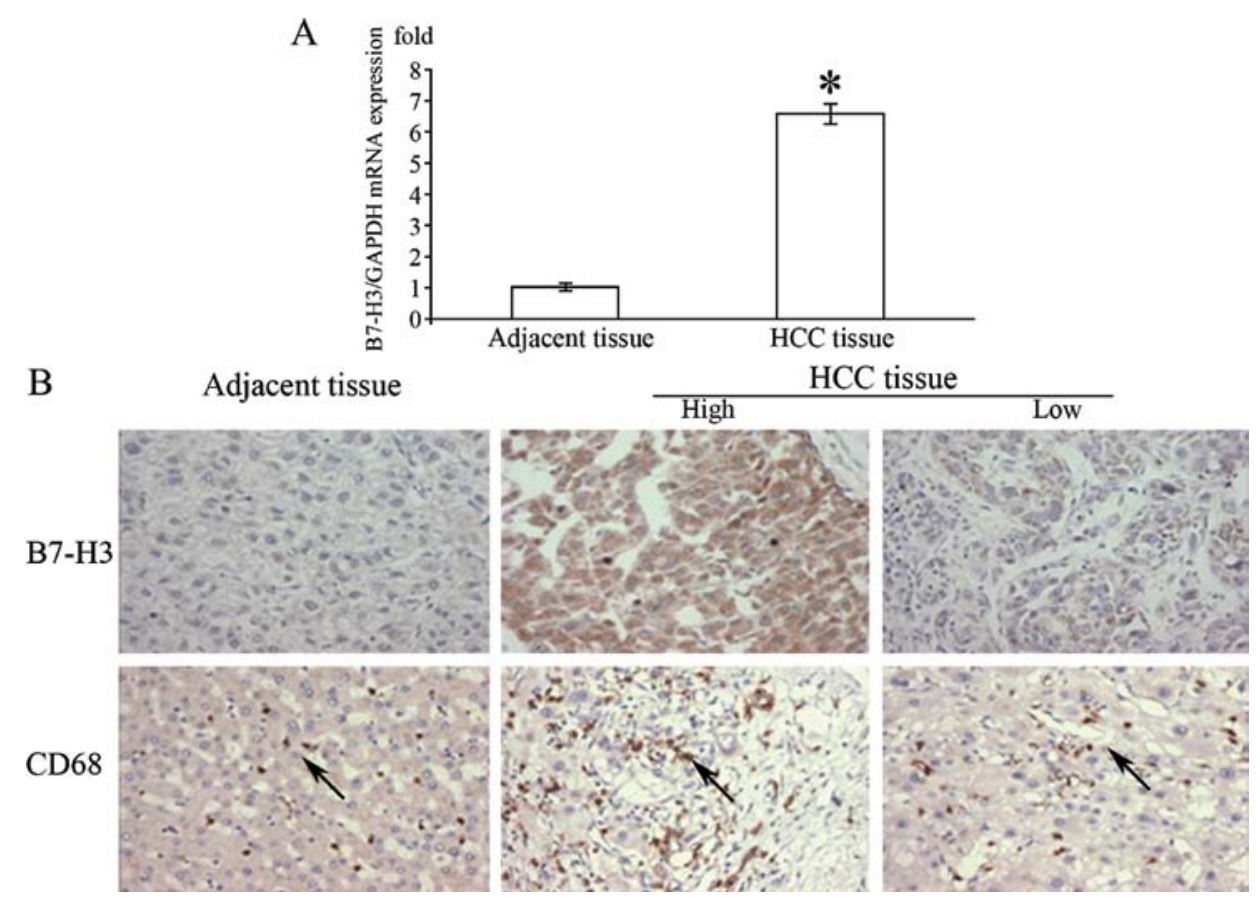

Figure 1. Expression of B7-H3 and CD68 in HCC tissues. (A) RT-PCR analysis of the mRNA level of B7-H3 in hepatocellular carcinoma (HCC) and adjacent normal liver tissues (samples from 20 human HCC tissues and the healthy adjacent tissue). GAPDH was a normalization control. " p $<0.05$ vs. adjacent normal liver tissues. (B) Immunohistochemical staining of B7-H3 or macrophage marker CD68 expression in HCC and adjacent normal liver tissues. Arrow indicates CD68 ${ }^{+}$TAM.

at $5 \times 10^{5}$ cells/well into the upper insert and cocultured with PMA-treated THP-1 macrophage cells without direct contact. In the coculture system, HepG 2 cells were cocultured with THP-1-differentiated macrophages for 24 and $48 \mathrm{~h}$ and were then harvested for use in the subsequent experiments.

RT-PCR analysis. Total cellular RNA was extracted for RT-PCR as previously described (25). Primers included were the following: B7-H3 (sense, 5'-ctctccaaaggaaagcgaggtgg acat-3' and antisense, 5'-agactgtacactgtaggtgctgaaatca-3'); HLA-DR (sense, 5'-tcacgtggcttcgaaatgga-3' and antisense, 5'-tccacctgcagtcgtaaac-3'); iNOS (sense, 5'-tccaaggtatcct ggagcga-3' and antisense, 5'-cagggacgggaactcctcta-3'); TNF- $\alpha$ (sense, 5'-ctgggcaggtctactttggg-3' and antisense, 5'-ctggaggccc cagtttgaat-3'); arginase 1 (Arg1) (sense, 5'-acggaagaatcagcctg gtg-3' and antisense, 5'-gtccacgtctctcaagccaa-3'); macrophagederived chemokine (CCL22) (sense, 5'-gcctactctgatgaccgtgg-3' and antisense, 5'-agagagttggcacaggcttc-3'); vascular endothelial cell growth factor (VEGF) (sense, 5'-tgcggatcaaacctca ccaa-3' and antisense, 5'-ctccagggcattagacagca-3'). The housekeeping gene GAPDH was used as the PCR control. RT-PCR products were analyzed via $1.5 \%$ agarose gel electrophoresis and stained with ethidium bromide for visualization using ultraviolet light.

Western blot assay. THP-1 cells were washed with PBS twice and lysed with $1 \mathrm{ml}$ RIPA lysis buffer containing a protease and phosphatase inhibitor for $30 \mathrm{~min}$ on ice. After removing the insoluble material by $12,000 \mathrm{x}$ g centrifugation for $30 \mathrm{~min}$ at $4^{\circ} \mathrm{C}$, the supernatants were collected. Cell lysate protein content was determined using a bicinchoninic acid (BCA) protein assay kit. Equivalent amounts of whole cell extracts were subjected to SDS-PAGE and transferred to PVDF membranes. The membranes were blocked with 5\% non-fat milk for $2 \mathrm{~h}$ and then incubated with the respective primary antibody overnight at $4^{\circ} \mathrm{C}$ followed by incubation with the appropriate HRP-conjugated secondary antibody for $2 \mathrm{~h}$ at room temperature. Blots were visualized with an ECL detection kit (Pierce, USA) and GAPDH was used as a loading control.

Short interfering RNA experiments. Short interfering RNA (siRNA) targeting human B7-H3 and the control scrambled siRNA were purchased from OriGene Company. Transfection was carried out by Lipofectamine 2000 (Invitrogen). Twentyfour and $48 \mathrm{~h}$ after transfection, HepG2 cells were cocultured with THP-1-differentiated macrophages for $12 \mathrm{~h}$ and then harvested for later RT-PCR analysis.

Inhibition of mitogen-activated protein kinase (MAPK) and signal transducer and activator of transcription 3 (STAT3) signaling pathways. For the inhibition of the MAPK pathway, HepG2 cells were treated with SB203580, PD98059 and SP600125, which are specific inhibitors of p38, ERK and JNK, respectively (Sigma) at $15 \mathrm{ng} / \mathrm{ml}$ for $2 \mathrm{~h}$. After a thorough wash to remove all the inhibitors, HepG2 cells were cocultured with THP-1-differentiated macrophages for $12 \mathrm{~h}$ and then harvested for later use. For the inhibition of the STAT3 pathway, HepG2 cells were treated with Tyrphostin AG490 (Sigma) at $20 \mathrm{ng} /$ $\mathrm{ml}$ for $24 \mathrm{~h}$. After a thorough wash to remove all the AG490, HepG2 cells were cocultured with THP-1-induced macrophages for $12 \mathrm{~h}$ and then harvested for later use.

\section{Results}

B7-H3 overexpression is associated with TAM infiltration in HCC tissues. As illustrated in Fig. 1A, the mRNA level of B7-H3 in the HCC tissues was significantly higher than 

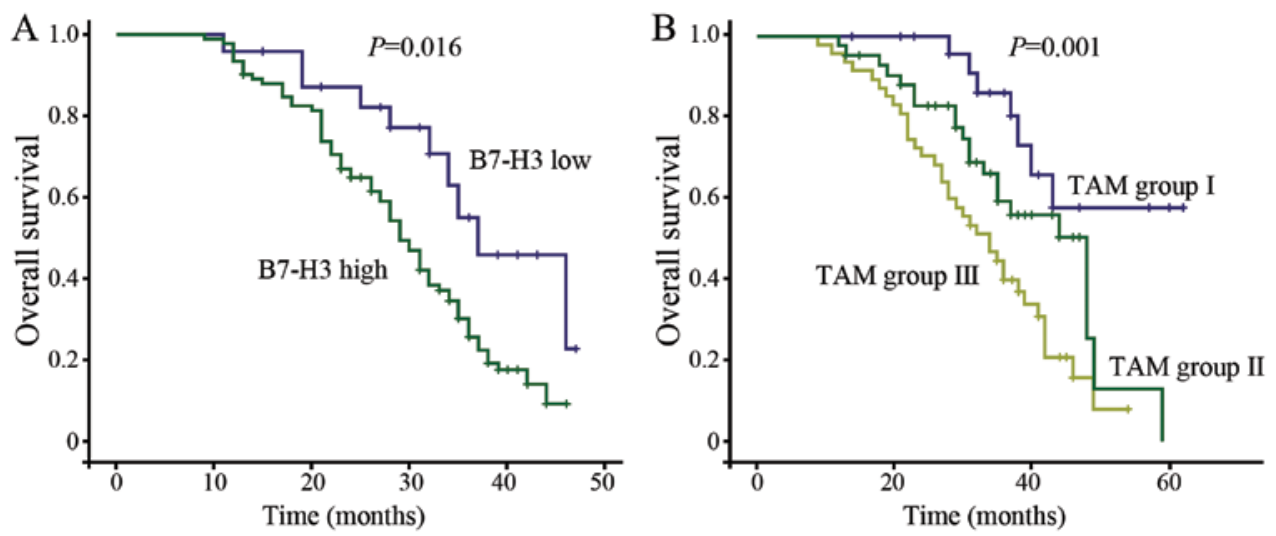

Figure 2. Kaplan-Meier survival analysis of the correlation between survival time and the (A) expression of B7-H3 and (B) the number of infiltrating macrophages labeled with CD68. B7-H3, B7 homologue 3.
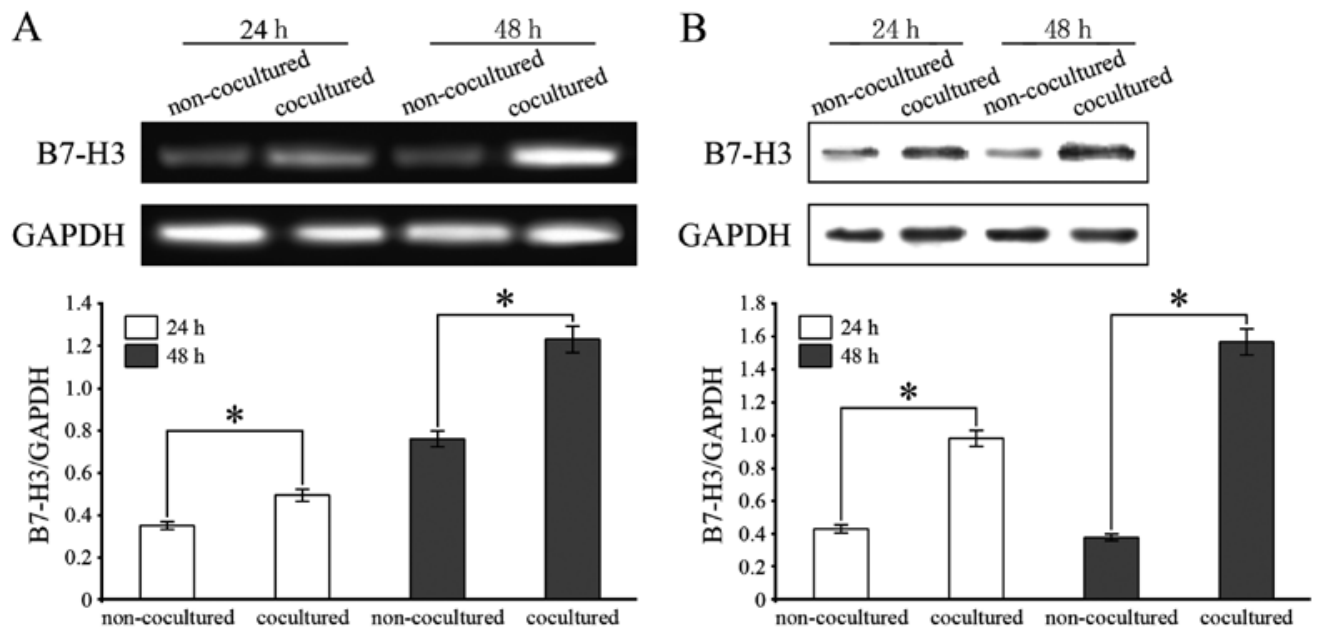

Figure 3. B7-H3 expression at the (A) mRNA and (B) protein level was upregulated in the THP-1 cells after coculture with HepG2 cells. B7-H3, B7 homologue 3 .

that in the adjacent normal tissues of the same patients. Immunohistochemical results showed that B7-H3 was overexpressed in the majority of cases in contrast to the normal liver cells, which appeared to be extremely diffuse in the tumor cell membrane, cytoplasm or both (Fig. 1B). Depending on the area of positive immunoreactivity, a final overall score (high tumor B7-H3 or low tumor B7-H3 expression) was established as described in Materials and methods. Of the 116 cases, $79.3 \%$ showed high expression of $\mathrm{B} 7-\mathrm{H} 3$ and $20.1 \%$ displayed a low degree of B7-H3 expression. In order to explore the potential role of B7-H3 in the tumor microenvironment, we analyzed the number of infiltrating TAMs. Among all HCC specimens, the number of infiltrating TAMs was significantly higher in the cancer than that in the normal tissue samples (Table I). Moreover, the number of infiltrating CD68 ${ }^{+}$TAMs was significantly higher in the $\mathrm{HCC}$ tissues with high $\mathrm{B} 7-\mathrm{H} 3$ expression than in the HCC tissues with weak expression; the mean number of infiltrating macrophages being $243.1 \pm 12.8$ and $87.3 \pm 5.9$, respectively.

B7-H3 overexpression and infiltrating TAMs predict poor outcome of HCC patients. Our previous results showed that the B7-H3 expression level was correlated with the number of TAMs in the evaluated HCC tissues. We then ascertained whether these two immunological factors could contribute to the outcome of the HCC patients. Kaplan-Meier survival analysis was used to examine the relationship between the B7-H3 expression level, the number of TAMs labeled with CD68 and their correlation with patient survival. The results showed that the overall survival time was significantly shorter in the high B7-H3 expression group than the overall survival time in the low B7-H3 expression group (risk ratio, 0.44; 95\% CI, 0.225-0.858; $\mathrm{p}=0.016$, Fig. 2A). A shorter overall survival time was also associated with a higher number of infiltrating TAMs (Fig. 2B). Tumor cells that expressed higher levels of B7-H3 exhibited higher levels of TAM infiltration $(\mathrm{p}<0.001$, Mann-Whitney test).

B7-H3 is upregulated on macrophages induced by PMA after coculture with HCC cells. To determine whether human HCC cells could induce the expression of B7-H3 on TAMs, we evaluated B7-H3 expression at the mRNA and protein levels in PMA-treated THP-1 cells cocultured with HepG2 cells by RT-PCR and western blot assays. B7-H3 mRNA expression was significantly upregulated in the THP-1 cells cocultured with HepG2 cells for 24 and 48 h (Fig. 3A). In addition, B7-H3 
Table I. Relationship between B7-H3 or TAM infiltration and the clinicopathological parameters of the HCC cases.

\begin{tabular}{|c|c|c|c|c|c|c|c|c|c|}
\hline \multirow[b]{2}{*}{ Parameters } & \multirow{2}{*}{$\begin{array}{l}\text { Total no. } \\
\text { of cases }\end{array}$} & \multicolumn{2}{|c|}{ B7-H3 expression } & \multirow[b]{2}{*}{$\mathrm{P}$-value } & \multirow{2}{*}{$\begin{array}{l}\text { Total no. } \\
\text { of cases }\end{array}$} & \multicolumn{3}{|c|}{ TAM infiltration } & \multirow[b]{2}{*}{ P-value } \\
\hline & & Low & High & & & $\mathrm{I}$ & II & III & \\
\hline Age (years) & & & & 0.571 & & & & & 0.285 \\
\hline$\leq 50$ & 43 & 9 & 34 & & 43 & 9 & 21 & 13 & \\
\hline$>50$ & 73 & 15 & 58 & & 73 & 12 & 28 & 33 & \\
\hline Gender & & & & 0.481 & & & & & 0.808 \\
\hline Male & 84 & 16 & 68 & & 84 & 14 & 36 & 34 & \\
\hline Female & 32 & 8 & 24 & & 32 & 7 & 13 & 12 & \\
\hline Vascular invasion & & & & 0.001 & & & & & 0.015 \\
\hline+ & 54 & 3 & 51 & & 54 & 5 & 21 & 28 & \\
\hline- & 62 & 21 & 41 & & 62 & 16 & 28 & 18 & \\
\hline TNM stage & & & & 0.038 & & & & & 0.036 \\
\hline I-II & 77 & 20 & 57 & & 77 & 18 & 34 & 25 & \\
\hline III-IV & 39 & 4 & 35 & & 39 & 3 & 15 & 21 & \\
\hline Lymph metastasis & & & & 0.041 & & & & & 0.034 \\
\hline+ & 32 & 3 & 29 & & 32 & 2 & 12 & 18 & \\
\hline- & 84 & 21 & 63 & & 84 & 19 & 37 & 28 & \\
\hline Tumor size $(\mathrm{cm})$ & & & & 0.014 & & & & & 0.298 \\
\hline$\leq 5$ & 77 & 21 & 56 & & 77 & 17 & 31 & 29 & \\
\hline$>5$ & 39 & 3 & 36 & & 39 & 4 & 18 & 17 & \\
\hline Tumor encapsulation & & & & 0.086 & & & & & 0.323 \\
\hline+ & 18 & 1 & 17 & & 18 & 2 & 9 & 7 & \\
\hline- & 98 & 23 & 75 & & 98 & 19 & 40 & 39 & \\
\hline Microsatellite tumors & & & & 0.023 & & & & & 0.036 \\
\hline Yes & 31 & 2 & 29 & & 31 & 2 & 13 & 16 & \\
\hline No & 85 & 22 & 63 & & 85 & 19 & 36 & 30 & \\
\hline Child-Pugh classification & & & & 0.789 & & & & & 0.624 \\
\hline A & 60 & 13 & 47 & & 60 & 11 & 27 & 22 & \\
\hline $\mathrm{B}$ & 56 & 11 & 45 & & 56 & 10 & 22 & 24 & \\
\hline Liver cirrhosis & & & & 0.203 & & & & & 0.83 \\
\hline Weak & 24 & 8 & 16 & & 24 & 4 & 9 & 11 & \\
\hline Moderate & 58 & 10 & 48 & & 58 & 12 & 26 & 20 & \\
\hline Strong & 34 & 6 & 28 & & 34 & 5 & 14 & 15 & \\
\hline HBsAg & & & & 0.142 & & & & & 0.212 \\
\hline+ & 76 & 13 & 63 & & 76 & 11 & 36 & 29 & \\
\hline- & 40 & 11 & 29 & & 40 & 10 & 13 & 17 & \\
\hline AFP & & & & 0.242 & & & & & 0.147 \\
\hline$\leq 200$ & 48 & 8 & 40 & & 48 & 9 & 25 & 14 & \\
\hline$>200$ & 67 & 16 & 51 & & 67 & 12 & 24 & 31 & \\
\hline Transfusion & & & & 0.286 & & & & & 0.071 \\
\hline+ & 47 & 8 & 39 & & 47 & 5 & 18 & 24 & \\
\hline- & 69 & 16 & 53 & & 69 & 16 & 31 & 22 & \\
\hline
\end{tabular}

Values in bold indicate statistical significance at $\mathrm{p}<0.05$. B7-H3, B7 homologue 3; TAMs, tumor-associated macrophages; HCC, hepatocellular carcinoma; TNM, tumor-node-metastasis; AFP, $\alpha$-fetoprotein; HBsAg, hepatitis B virus surface antigen.

protein expression was also increased in the TAMs after 24 and 48 h of coculture with HCC cells (Fig. 3B). In addi- tion, monocytes were isolated from human peripheral blood and induced to macrophages by PMA. The B7-H3 mRNA and 

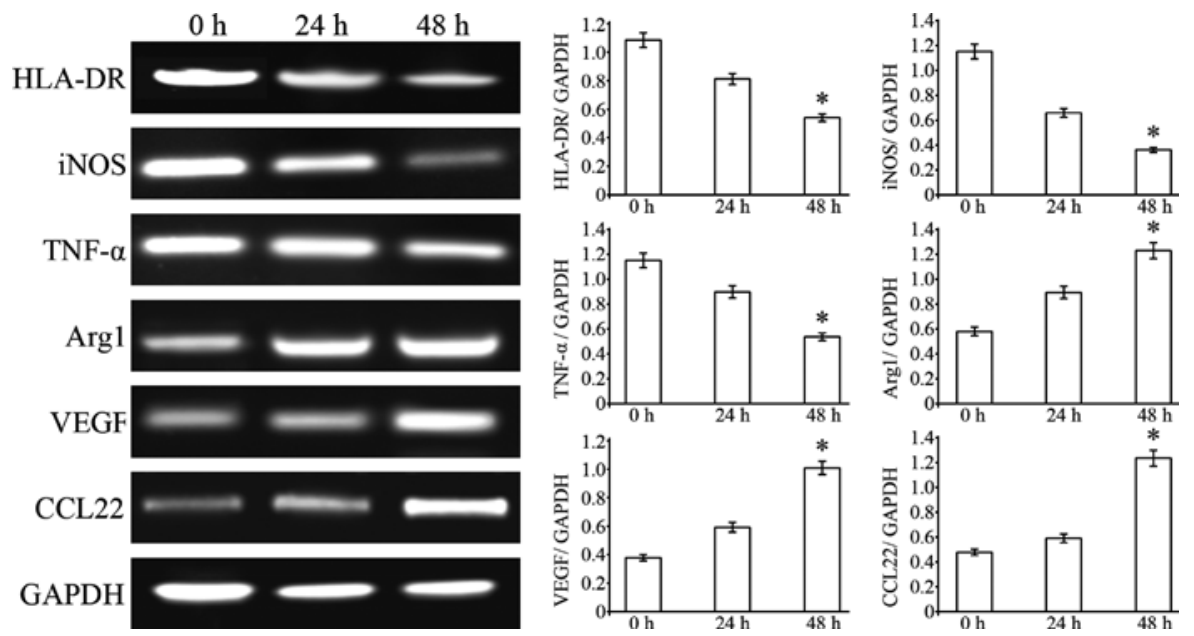

Figure 4. Expression of Arg1, VEGF and CCL22 was upregulated in the THP-1 cells after coculture with HepG2 cells, while the expression of HLA-DR, iNOS and TNF- $\alpha$ was reduced.
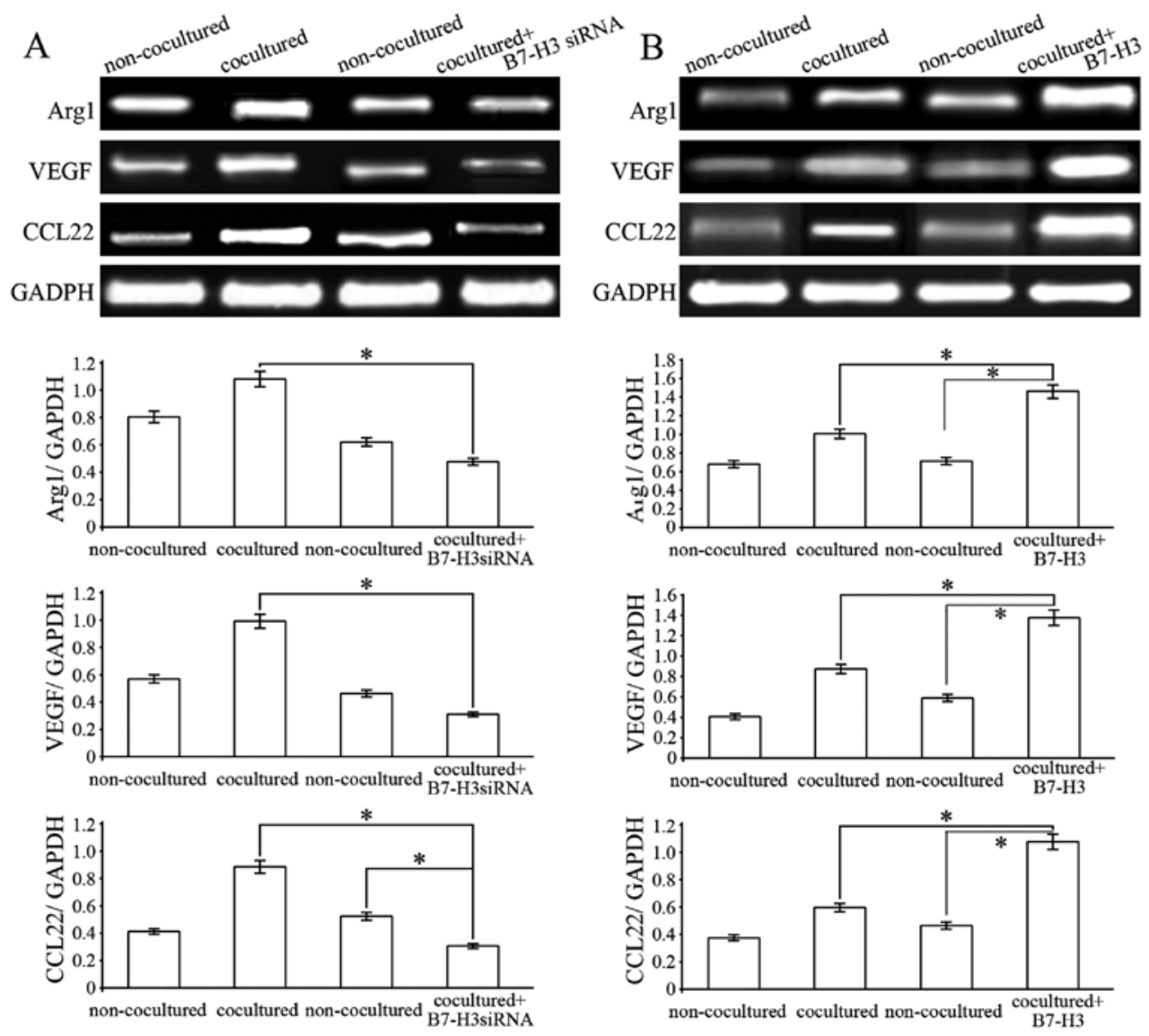

Figure 5. (A) Expression of Arg1, VEGF and CCL22 was reduced in the THP-1 cells after coculture with B7-H3 siRNA-treated HepG2 cells, (B) while upregulated after coculture with B7-H3-treated HepG2 cells.

its protein expression in macrophages cocultured with HepG2 cell lines was significantly upregulated (data not shown).

B7-H3 promotes M2 phenotype macrophage polarization. PMA-induced THP-1 cells have been described as 'innate' macrophages that can differentiate into M1 or M2 macrophages (26). To determine whether HepG2-secreted B7-H3 can influence THP-1 cell differentiation into different macrophage subtypes, we examined the expression of M1 and M2 macro- phage markers by RT-PCR assays. The results showed that HLA-DR, iNOS and TNF- $\alpha$ mRNA levels in the THP-1 cells were significantly reduced after 24 and $48 \mathrm{~h}$ of coculturing with HepG2 cells. However, Arg1, CCL22 and VEGF mRNA expression levels in the THP-1 cells were significantly increased after 24 and 48 h of coculturing with the HepG2 cells (Fig. 4). The results suggest that hepatoma cells can promote THP-1-differentiated macrophages towards M2 phenotype polarization. 

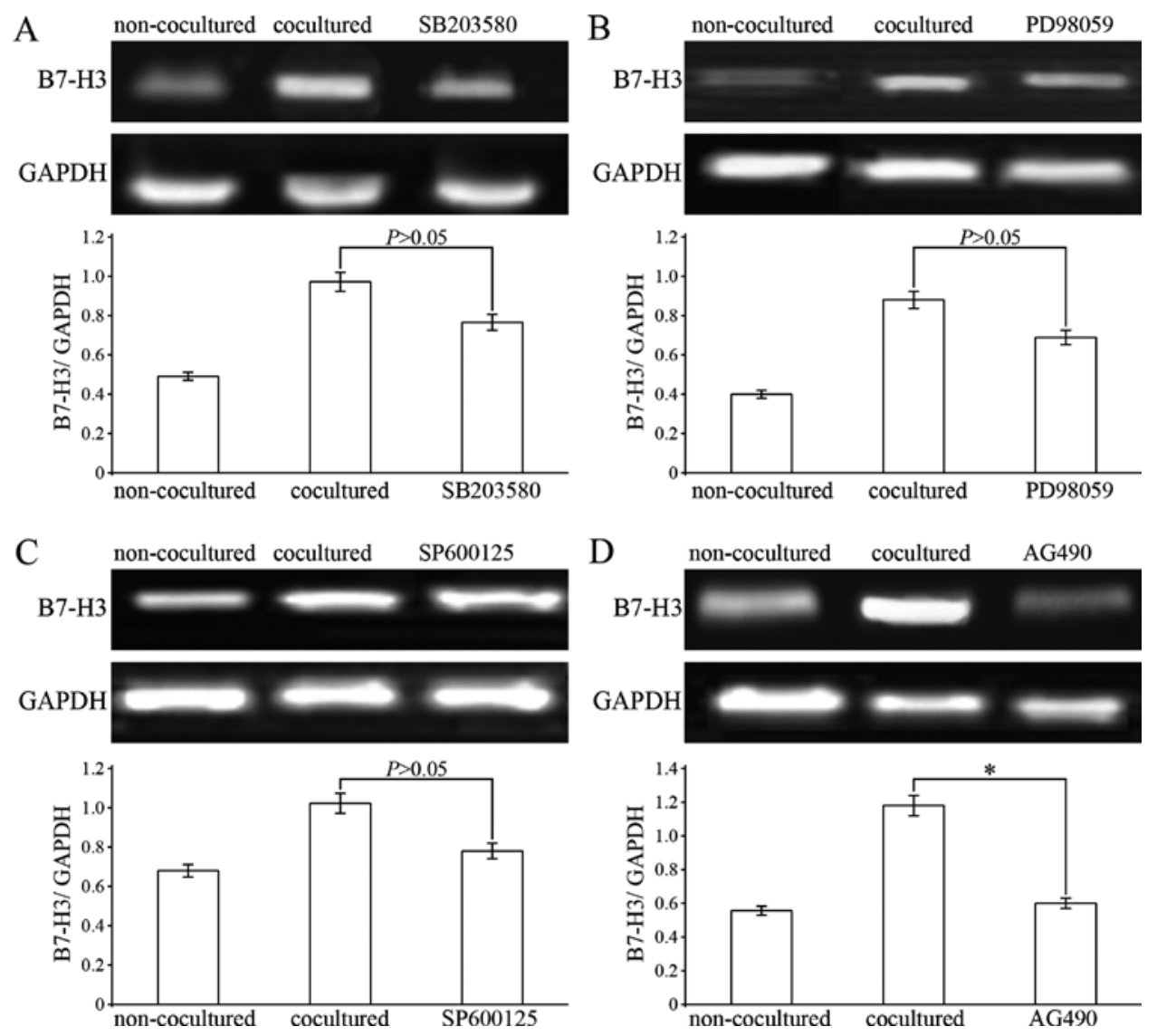

Figure 6. Induction of B7-H3 expression in the THP-1 cells was inhibited partially by blocking STAT3 pathway. HepG2 cells were pretreated with (A) SB203580, (B) PD98059, (C) SB203580 and (D) AG490, then coculture with PMA-treated THP-1 cells. B7-H3, B7 homologue 3; PMA, phorbol 12-myristate 13-acetate.

Blocking of B7-H3 interferes with the hepatoma cell-mediated M2 macrophage polarization. To determine whether B7-H3 is involved in promoting THP-1-mediated macrophage differentiation into M2 macrophages, we pretreated HepG2 cells with B7-H3 siRNA and then examined the expression of M2 macrophage markers. The results showed that Arg1, CCL22 and VEGF mRNA expression levels in the THP-1 cells were significantly decreased after coculturing with B7-H3-knockdown HepG2 cells, compared with the control group. This suggests that pretreatment with $\mathrm{B} 7-\mathrm{H} 3$ siRNA abolished B7-H3-promoted M2 marker expression in the THP-1 cells (Fig. 5A). In contrast, pretreatment with B7-H3 $(30 \mathrm{ng} / \mathrm{ml})$ markedly increased the expression of M2 macrophage markers (Fig. 5B). Taken together, these results showed that HepG2-secreted B7-H3 could polarize THP-1differentiated macrophages to an M2 macrophage phenotype. This may lead to the alteration of proinflammatory cytokine expression pattern, thus leading to suppression of the immune response within the tumor microenvironment.

Upregulation of B7-H3 expression on THP-1 cells is inhibited by partial blocking of the STAT3 signaling pathway. To further clarify the mechanism of upregulation of B7-H3 expression on THP-1-differentiated macrophages cocultured with HCC cells, experiments of the blocking of the MAPK (p38, ERK and JNK) and STAT3 signaling pathways were performed. After pretreatment with SB203580, PD98059 and SP600125, THP-1-induced macrophages were cocultured with HepG2 cells for $6 \mathrm{~h}$ and then harvested for detection of B7-H3 mRNA expression. The results showed that SB203580, PD98059 and SP600125 had the tendency to abrogate the upregulation of B7-H3 mRNA expression in THP-1-induced macrophages, however, the data were not statistically significant (Fig. 6A-C). As for the STAT3 pathway, we chose Tyrphostin AG490 as its specific inhibitor. AG490 partially inhibited the increase in B7-H3 mRNA expression in the THP-1-induced macrophages cocultured with the HepG2 cells (Fig. 6D). These results suggest that upregulation of $\mathrm{B} 7-\mathrm{H} 3$ expression on THP-1-differentiated macrophages induced by the HCC cell microenvironment may be via the STAT3 signaling pathway.

\section{Discussion}

As HCC has been shown to be immunogenic, immunotherapy is an alternative promising therapeutic approach (27). Immunotherapy aims to provide a more efficient way to target tumor cells by inducing or enhancing the existing tumorspecific immune response. However, HCC demonstrated potential immunoresistance in the local tumor microenvironment allowing the tumor to evade a cytotoxic response (28). Aberrant regulation of immune-stimulating antigens is one of the several complicated mechanisms concerning tumor immunoescape $(29,30)$. In the present study, we showed that B7-H3 was uniformly overexpressed in hepatoma cells. Increased $\mathrm{B} 7-\mathrm{H} 3$ expression was detected in $79.3 \%$ of the $61 \mathrm{HCC}$ specimens examined. Additionally, B7-H3 expression 
was significantly correlated with the patient overall survival time; that is lower levels of B7-H3 expression were associated with a prolonged survival time. In light of previous studies and the results of the present study $(9,10,14)$, it appears that B7-H3 plays a critical role in the pathogenesis and development of HCC, however, its exact role remains unclear.

Several studies have demonstrated that TAMs play a key role in the tumor progression of HCC $(18,31)$. TAMs are mainly polarized towards the $\mathrm{M} 2$ phenotype and favors tumor formation and progression $(32,33)$. In order to explore the mechanisms underlying the overexpression of B7-H3, we analyzed the relationship between B7-H3 expression and TAMs in HCC. Immunohistochemical results showed that the B7-H3 expression in tumor cells was correlated with the infiltration of TAMs in HCC tissues, and the number of TAMs had a negative correlation with the patient survival time. Moreover, HCC cells upregulated B7-H3 expression in PMA-induced THP-1 cells. These findings suggest that the macrophages that infiltrate the HCC tissues may be important for promoting tumor progression, and B7-H3 may be involved in this process. Our results are in line with previous similar studies of other costimulatory molecules that function in TAM regulation. For example, Chen et al showed that TAMs could be induced to express the B7-H3 molecule in tumor stroma when cultured with tumor cells in lung cancer. Upregulation of B7-H3 on TAMs is a major pro-inflammatory resource and novel immune escape mechanism in the tumor milieu (34).

TAMs are the prominent population of infiltrating leukocytes and the major source of inflammatory cytokines in the tumor milieu $(35,36)$. Upon activation, macrophages can release a vast diversity of cytokines, proteolytic enzymes, growth factors and inflammatory mediators that may directly influence the behavior of tumor cells (37). Generally, the M1 phenotype could secrete reactive oxygen and nitrogen intermediates to kill cancer cells, and immunomodulatory factors including TNF- $\alpha$ and interleukin-1 $\beta$ (IL-1 $\beta$ ) to recruit CTL cells to attack cancer (38). The M2 phenotype has the opposite effects. They release vascular endothelial cell growth factor (VEGF), platelet-derived growth factor (PDGF), tumor transforming growth factor (TGF)- $\beta$ and IL-10 that promote cancer cell growth. Moreover, these M2-like TAMs can produce a variety of matrix metalloproteinases and chemokines, such as MMP-2, MMP-7, MMP-9, CCL18, CCL22 that facilitate cancer micrometastasis $(39,40)$. In the present study, the coculture experiment revealed that M2 phenotype marker expression was significantly increased on the PMA-treated THP-1 cells. Accordingly, M1 phenotype marker expression was reduced vice versa. Therefore, we concluded that the subtle cytokine changes in the HCC microenvironment have the potential to promote TAMs to differentiate into the M2 phenotype, and overexpression of B7-H3 may be involved in this.

Accumulating data revealed that B7 costimulatory molecules were highly expressed on TAMs and these TAMs expressing certain B7 molecules favored pro-inflammatory response to immune tolerance in the tumor milieu. Chen et al demonstrated that B7-H4-expressing macrophages were related to tumor size, lymph node metastasis and TNM stage, which may promote tumor progression (41). In addition, B7-H1 and B7-H4 have been found to be involved in the shift from inflammatory M1 macrophage to the anti-inflammatory
M2-like macrophage differentiation $(42,43)$. In the present study, the macrophages derived from the THP-1 cells cocultured with siB7-H3-treated HepG2 cells reduced expression levels of Arg1, CCL22 and VEGF, which are distinctive of M2 macrophages, demonstrating that the B7-H3 signaling pathway significantly interfered with the switching of the macrophage phenotype towards M2. Based on the results collected, we can speculate that the complex of the HCC tumor microenvironment resulted in the aberrant expression of tumor-associated molecules including B7-H3, which further accelerated the tumor progression and could be released into the blood as its soluble form (sB7-H3). sB7-H3 with other immunosuppressive cytokines, such as IL-10, TGF- $\beta$ and IL- $1 \beta$, promote the polarization of TAMs towards the M2 phenotype. Therefore, we hypothesized that B7-H3 may also function as a chemotactic factor, attracting monocytes in the peripheral blood to migrate to tumor tissues and induce the differentiation of macrophages, thereby promoting tumor oncogenesis and development.

Recent studies have demonstrated that the expression of the B7-H3 gene is involved in activation of the STAT3 and MAPK pathways $(44,45)$. Moreover, several major signaling pathways and their modulators/targets, including the MAPK and STAT signaling pathways, are involved in directing the macrophage plasticity and polarized function, and are associated with reciprocal skewing of macrophage polarization between the M1 and M2 states $(46,47)$. In the present study, the upregulation of M2-phenotype marker expression in the THP-1-differentiated macrophages was partially abrogated by inhibitors specific for STAT3 signaling, while no obvious effects were noted by the p38, JNK and ERK specific inhibitors. Therefore, the M2-like TAMs in HCC tissues may be induced by the inflammatory cytokines released from HCC tissues through activating the B7-H3/STAT3 signaling pathway.

In summary, the present study revealed that overexpression of B7-H3 in tumor cells was associated with TAM infiltration in HCC tissues, and B7-H3 expression was induced on the surface of PMA-induced macrophages facilitating M2-TAM polarization in the HCC microenvironment. In support, inflammatory cytokines released from M2-TAMs stimulated tumor growth and metastasis. Furthermore, the B7-H3/STAT3 signaling pathway may be involved in switching macrophages to the M2 phenotype and the negative regulation of the T lymphocytemediated immune response. Therefore, future studies to identify methods of inhibiting B7-H3 expression in HCC are warranted. TAM-tumor cell interaction-induced B7-H3 represents a novel immune escape target in the $\mathrm{HCC}$ tumor milieu.

\section{Acknowledgements}

This study was supported by the National Natural Science Foundation of China (81402228), HeBei Province Medical Foundation (ZL20140334) and HeBei Province Education Foundation (QN2014049).

\section{References}

1. Flores A and Marrero JA: Emerging trends in hepatocellular carcinoma: focus on diagnosis and therapeutics. Clin Med Insights Oncol 8: 71-76, 2014

2. Abdel-Rahman O: Systemic therapy for hepatocellular carcinoma (HCC): from bench to bedside. J Egypt Natl Canc Inst 25: 165-171, 2013. 
3. Salomao M, Remotti H, Vaughan R, Siegel AB, Lefkowitch JH and Moreira RK: The steatohepatitic variant of hepatocellular carcinoma and its association with underlying steatohepatitis. Hum Pathol 43: 737-746, 2012.

4. Forner A, Llovet JM and Bruix J: Hepatocellular carcinoma. Lancet 379: 1245-1255, 2012.

5. Rossi L, Zoratto F, Papa A, et al: Current approach in the treatment of hepatocellular carcinoma. World J Gastrointest Oncol 2: 348-359, 2010.

6. Palmer DH, Midgley RS, Mirza N, et al: A phase II study of adoptive immunotherapy using dendritic cells pulsed with tumor lysate in patients with hepatocellular carcinoma. Hepatology 49: 124-132, 2009.

7. Hernandez-Gea V, Alsinet C and Llovet JM: Oncolytic immunotherapeutic virus in HCC: can it compete with molecular therapies? J Hepatol 59: 882-884, 2013.

8. Ceeraz S, Nowak EC and Noelle RJ: B7 family checkpoint regulators in immune regulation and disease. Trends Immunol 34 556-563, 2013.

9. Sun TW, Gao Q, Qiu SJ, et al: B7-H3 is expressed in human hepatocellular carcinoma and is associated with tumor aggressiveness and postoperative recurrence. Cancer Immunol Immunother 61 2171-2182, 2012.

10. Wang F, Wang G, Liu T, Yu G, Zhang G and Luan X: B7-H3 was highly expressed in human primary hepatocellular carcinoma and promoted tumor progression. Cancer Invest 32: 262-271, 2014.

11. Geng L, Deng J, Jiang G, et al: B7-H1 up-regulated expression in human hepatocellular carcinoma tissue: correlation with tumor interleukin-10 levels. Hepatogastroenterology 58: 960-964, 2011

12. Wang L, Kang FB and Shan BE: B7-H3-mediated tumor immunology: friend or foe? Int J Cancer 134: 2764-2771, 2014.

13. Chapoval AI, Ni J, Lau JS, et al: B7-H3: a costimulatory molecule for $\mathrm{T}$ cell activation and IFN- $\gamma$ production. Nat Immunol 2 : 269-274, 2001

14. Chen W, Liu P, Wang Y, et al: Characterization of a soluble B7-H3 (sB7-H3) spliced from the intron and analysis of sB7-H3 in the sera of patients with hepatocellular carcinoma. PLoS One 8: e76965, 2013.

15. Goubran HA, Kotb RR, Stakiw J, Emara ME and Burnouf T: Regulation of tumor growth and metastasis: the role of tumor microenvironment. Cancer Growth Metastasis 7: 9-18, 2014.

16. Santoni M, Massari F, Amantini C, et al: Emerging role of tumor-associated macrophages as therapeutic targets in patients with metastatic renal cell carcinoma. Cancer Immuno Immunother 62: 1757-1768, 2013.

17. Galdiero MR, Bonavita E, Barajon I, Garlanda C, Mantovani A and Jaillon S: Tumor associated macrophages and neutrophils in cancer. Immunobiology 218: 1402-1410, 2013.

18. Shirabe K, Mano Y, Muto J, et al: Role of tumor-associated macrophages in the progression of hepatocellular carcinoma. Surg Today 42: 1-7, 2012.

19. Fan QM, Jing YY, Yu GF, et al: Tumor-associated macrophages promote cancer stem cell-like properties via transforming growth factor-beta1-induced epithelial-mesenchymal transition in hepatocellular carcinoma. Cancer Lett 352: 160-168, 2014

20. Wang L,Zhang Q, Chen W, et al: B7-H3 is overexpressed in patients suffering osteosarcoma and associated with tumor aggressiveness and metastasis. PLoS One 8: e70689, 2013.

21. Liu H, Tekle C, Chen YW, et al: B7-H3 silencing increases paclitaxel sensitivity by abrogating Jak2/Stat3 phosphorylation. Mol Cancer Ther 10: 960-971, 2011.

22. Sun J, Mao Y, Zhang YQ, et al: Clinical significance of the induction of macrophage differentiation by the costimulatory molecule B7-H3 in human non-small cell lung cancer. Oncol Lett 6: 1253-1260, 2013.

23. Zhang G, Wang J, Kelly J, et al: B7-H3 augments the inflammatory response and is associated with human sepsis. J Immunol 185: 3677-3684, 2010.

24. Tjiu JW, Chen JS, Shun CT, et al: Tumor-associated macrophageinduced invasion and angiogenesis of human basal cell carcinoma cells by cyclooxygenase-2 induction. J Invest Dermatol 129: 1016-1025, 2009.

25. Wang L, Kang F, Li J, Zhang J and Shan B: Overexpression of p65 attenuates celecoxib-induced cell death in MDA-MB-231 human breast cancer cell line. Cancer Cell Int 13: 14, 2013.
26. Wu TH, Li YY, Wu TL, et al: Culture supernatants of different colon cancer cell lines induce specific phenotype switching and functional alteration of THP-1 cells. Cell Immunol 290: 107-115, 2014.

27. Kabilova TO, Kovtonyuk LV, Zonov EV, et al: Immunotherapy of hepatocellular carcinoma with small double-stranded RNA. BMC Cancer 14: 338, 2014.

28. Schmidt N, Neumann-Haefelin C and Thimme R: Cellular immune responses to hepatocellular carcinoma: lessons for immunotherapy. Dig Dis 30: 483-491, 2012.

29. Seliger B and Quandt D: The expression, function, and clinical relevance of B7 family members in cancer. Cancer Immunol Immunother 61: 1327-1341, 2012.

30. Bottino C, Dondero A, Bellora F, et al: Natural killer cells and neuroblastoma: tumor recognition, escape mechanisms, and possible novel immunotherapeutic approaches. Front Immunol 5: 56, 2014.

31. Capece D, Fischietti M, Verzella D, et al: The inflammatory microenvironment in hepatocellular carcinoma: a pivotal role for tumor-associated macrophages. Biomed Res Int 2013: 187204, 2013.

32. Lievense LA, Bezemer K, Aerts JG and Hegmans JP: Tumorassociated macrophages in thoracic malignancies. Lung Cancer 80: 256-262, 2013

33. Martinez FO, Sica A, Mantovani A and Locati M: Macrophage activation and polarization. Front Biosci 13: 453-461, 2008.

34. Chen C, Shen Y, Qu QX, Chen XQ, Zhang XG and Huang JA: Induced expression of B7-H3 on the lung cancer cells and macrophages suppresses T-cell mediating anti-tumor immune response. Exp Cell Res 319: 96-102, 2013.

35. Lewis CE and Pollard JW: Distinct role of macrophages in different tumor microenvironments. Cancer Res 66: 605-612, 2006.

36. Yuan A, Chen JJ and Yang PC: Pathophysiology of tumorassociated macrophages. Adv Clin Chem 45: 199-223, 2008.

37. Yue ZQ, Liu YP, Ruan JS, Zhou L and Lu Y: Tumor-associated macrophages: a novel potential target for cancer treatment. Chin Med J 125: 3305-3311, 2012.

38. Wang $\mathrm{H}$, Wang $\mathrm{X}$, Li X, et al: $\mathrm{CD} 68^{+} \mathrm{HLA}^{-\mathrm{DR}^{+} \mathrm{M} 1-\text { like macro- }}$ phages promote motility of HCC cells via NF- $\kappa \mathrm{B} / \mathrm{FAK}$ pathway. Cancer Lett 345: 91-99, 2014

39. Lee JH, Lee GT, Woo SH, et al: BMP-6 in renal cell carcinoma promotes tumor proliferation through IL-10-dependent M2 polarization of tumor-associated macrophages. Cancer Res 73: 3604-3614, 2013

40. Ma YY, He XJ, Wang HJ, et al: Interaction of coagulation factors and tumor-associated macrophages mediates migration and invasion of gastric cancer. Cancer Sci 102: 336-342, 2011

41. Chen C, Zhu YB, Shen Y, Zhu YH, Zhang XG and Huang JA: Increase of circulating B7-H4-expressing $\mathrm{CD} 68^{+}$macrophage correlated with clinical stage of lung carcinomas. J Immunother 35: 354-358, 2012.

42. Chen J, Li G, Meng H, et al: Upregulation of B7-H1 expression is associated with macrophage infiltration in hepatocellular carcinomas. Cancer Immunol Immunother 61: 101-108, 2012.

43. Chen C, Qu QX, Shen Y, et al: Induced expression of B7-H4 on the surface of lung cancer cells by the tumor-associated macrophages: a potential mechanism of immune escape. Cancer Lett 317: 99-105, 2012.

44. Tekle C, Nygren MK, Chen YW, et al: B7-H3 contributes to the metastatic capacity of melanoma cells by modulation of known metastasis-associated genes. Int J Cancer 130: 2282-2290, 2012.

45. Chen X, Quinn EM, Ni H, et al: B7-H3 participates in the development of experimental pneumococcal meningitis by augmentation of the inflammatory response via a TLR2-dependent mechanism. J Immunol 189: 347-355, 2012.

46. Zhang W, Xu W and Xiong S: Macrophage differentiation and polarization via phosphatidylinositol 3-kinase/Akt-ERK signaling pathway conferred by serum amyloid P component. J Immunol 187: 1764-1777, 2011.

47. Zhou D, Huang C, Lin Z, et al: Macrophage polarization and function with emphasis on the evolving roles of coordinated regulation of cellular signaling pathways. Cell Signal 26: 192-197, 2014. 